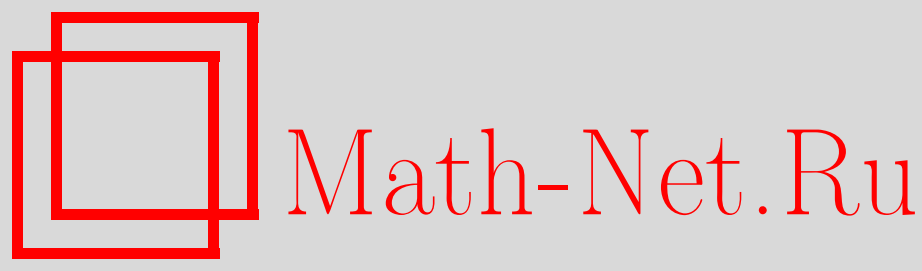

М. В. Иоффе, Е. В. Колеватова, Д. Н. Нишнианидзе, Некоторые свойства форминвариантной двумерной модели скарф II, ТMФ, 2015, том 185, номер 1, 99-108

DOI: https://doi.org/10.4213/tmf8912

Использование Общероссийского математического портала Math-Net.Ru подразумевает, что вы прочитали и согласны с пользовательским соглашением http://www . mathnet.ru/rus/agreement

Параметры загрузки:

IP : 54.198 .187 .58

26 апреля 2023 г., 08:49:50

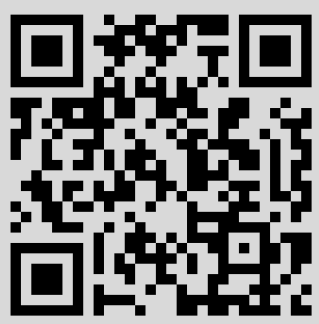




\title{
НЕКОТОРЫЕ СВОЙСТВА ФОРМИНВАРИАНТНОЙ ДВУМЕРНОЙ МОДЕЛИ СКАРФ ІІ
}

\begin{abstract}
В рамках суперсимметричной квантовой механики рассматривается двумерная квантовая модель Скарф II. Систематизируются ранее полученные для этой интегрируемой модели результаты и выводятся некоторые новые свойства. В частности, показано, что модель является точно или квазиточно решаемой в разных областях значений параметра системы. Обнаружено вырождение спектра для некоторых конкретных значений параметров. Вычислено действие операторов симметрии четвертого порядка по импульсам на произвольную волновую функцию, полученную с помощью двойной форминвариантности.
\end{abstract}

Ключевые слова: суперсимметрия, форминвариантность, интегрируемые модели, точная решаемость, соотношения сплетания.

DOI: $10.4213 / \operatorname{tmf} 8912$

\section{1. ВВЕДЕНИЕ}

Известны два обобщения суперсимметричного подхода [1] в квантовой механике от одномерного случая к двумерному. В первом из них суперзаряды линейны по производным, а соответствующий супергамильтониан включает в себя как пару скалярных компонент, так и матричную компоненту [2]. Матричная компонента может интерпретироваться, например, как гамильтониан Паули для нерелятивистского фермиона, двигающегося в плоскости во внешнем электромагнитном поле [3]. В противоположность этому, в альтернативном суперсимметричном подходе [4] с суперзарядами второго порядка по импульсам отсутствуют матричные компоненты в супергамильтониане. В данном методе основной начальный этап - решение суперсимметричных соотношений сплетания между парой скалярных шредингеровских гамильтонианов. Сплетающие операторы - суперзаряды второго порядка - классифицированы в соответствии с их метрикой по старшим производным [4]. Для простейшего случая суперзарядов с лоренцевой (гиперболической) метрикой все возможные решения для потенциалов были найдены явно [5].

* Санкт-Петербургский государственный университет, Санкт-Петербург, Россия. E-mail: m.ioffe@spbu.ru, e.v.krup@yandex.ru

${ }^{\dagger}$ Государственный университет им. Акаки Церетели, Кутаиси, Грузия. E-mail: cutaisi@yahoo.com 
В одномерном случае среди скалярных гамильтонианов-суперпартнеров существует подмножество с так называемым свойством форминвариантности [1], [6], [7]. Как правило, это свойство обеспечивает элегантный чисто алгебраический метод решения задачи, а именно позволяет аналитически находить и энергетический спектр, и соответствующие волновые функции. K классу форминвариантных потенциалов принадлежат [8] все известные точно решаемые одномерные потенциалы (некоторые дополнительные, неизвестные, точно решаемые системы были построены [9] как раз суперсимметричным методом). Понятие форминвариантности было также обобщено [10]-[13] и на двумерную суперсимметричную квантовую механику. При этом априори форминвариантность, вообще говоря, не обеспечивает решаемости системы, однако для некоторых моделей она, вместе с другими методами, помогает [10], [14]--[16] решить задачу точно или квазиточно [17].

Двумерные обобщения суперсимметричной квантовой механики обладают рядом свойств, которые для полноты формулировки должны еще быть изучены. Имеются в виду такие вопросы, как связь между точно и квазиточно решаемыми моделями с одним и тем же потенциалом, действие операторов симметрии на построенные волновые функции, особенно при наличии возможного вырождения спектра. Ниже показано, что последнее реализуется для некоторых конкретных значений константы связи. Именно этот круг вопросов исследован в настоящей работе для случая модели Скарф II - хронологически последней построенной решаемой модели, позволяющей применить все перечисленные выше методы.

Структура работы следующая. В разделе 2 формулируется двумерная модель Скарф II и представлены две формы специального суперсимметричного разделения переменных. Одна из них (см. п. 2.1) дает точную решаемость модели при дискретных отрицательных значениях $a_{k}=-k$ параметра $a$. В п. 2.2 показано, что другой метод суперсимметричного разделения работает для области значений параметра $a>-1 / 2$, которая не пересекается с предыдущей областью. В этом случае часть волновых функций получается посредством соотношений сплетания и форминвариантности. Детали, касающиеся возможной вырожденности энергетических уровней и действия операторов симметрии, рассматриваются в разделе 3. Показано, что все построенные волновые функции одновременно являются собственными функциями операторов симметрии.

\section{2. МОДЕЛЬ СКАРФ ІІ: ТОЧНАЯ И КВАЗИТОЧНАЯ РЕШАЕМОСТЬ}

Соотношения сплетания

$$
H^{(1)} Q^{+}=Q^{+} H^{(2)}, \quad Q^{-} H^{(1)}=H^{(2)} Q^{-}
$$

между парой гамильтонианов

$$
\begin{gathered}
H^{(i)}(\vec{x} ; a)=-\Delta^{(2)}+V^{(i)}(\vec{x} ; a), \quad i=1,2, \quad \vec{x}=\left(x_{1}, x_{2}\right), \\
\Delta^{(2)} \equiv \partial_{1}^{2}+\partial_{2}^{2}, \quad \partial_{i} \equiv \frac{\partial}{\partial x_{i}},
\end{gathered}
$$

играют главную роль в суперсимметричном подходе к квантовой механике. В случае лоренцевой метрики для старших производных в суперзаряде имеется [11] следую- 
щее решение для $Q^{ \pm}$:

$$
\begin{aligned}
Q^{+}=( & \left.Q^{-}\right)^{\dagger}=4 \partial_{+} \partial_{-}+2 a \operatorname{th} \frac{x_{+}}{2} \partial_{-}+2 a \operatorname{cth} \frac{x_{-}}{2} \partial_{+}+a^{2} \text { th } \frac{x_{+}}{2} \operatorname{cth} \frac{x_{-}}{2}- \\
& -\left(\operatorname{ch} x_{1}\right)^{-2}\left[c(2 b+1) \operatorname{sh} x_{1}+\left(c^{2}-b^{2}-b\right)\right]+ \\
& +\left(\operatorname{ch} x_{2}\right)^{-2}\left[c(2 b+1) \operatorname{sh} x_{2}+\left(c^{2}-b^{2}-b\right)\right]
\end{aligned}
$$

где $x_{ \pm} \equiv x_{1} \pm x_{2}, \partial_{ \pm}=\partial / \partial x_{ \pm}, a, b, c$ - вещественные параметры $(b, c>0)$, а соответствующие гамильтонианы-суперпартнеры являются двумерными обобщениями модели Скарф II:

$$
H^{(i)}(\vec{x} ; a)=-\Delta^{(2)}-\frac{a(a \mp 1)}{2}\left(\frac{1}{\operatorname{ch}^{2}\left(x_{+} / 2\right)}-\frac{1}{\operatorname{sh}^{2}\left(x_{-} / 2\right)}\right)+U\left(x_{1}\right)+U\left(x_{2}\right),
$$

где одномерный потенциал $U$ имеет вид известного потенциала модели Скарф II:

$$
U(x)=\frac{c(2 b+1) \operatorname{sh} x+\left(c^{2}-b^{2}-b\right)}{\operatorname{ch}^{2} x} .
$$

Здесь и ниже мы иногда для простоты опускаем явную зависимость от некоторых параметров, но будем ее восстанавливать по мере необходимости.

2.1. Точная решаемость. В обоих гамильтонианах $H^{(i)}$ имеется смешанный член, который не позволяет провести стандартную процедуру разделения переменных. Однако структура этого слагаемого позволяет использовать специальную процедуру - суперсимметричное разделение переменных [13]. Выбирая [18] специальное значение параметра $a=-1$, получаем, что один из гамильтонианов-суперпартнеров $\left(H^{(2)}\right)$ допускает стандартное разделение переменных. Более того, одномерные уравнения Шредингера с потенциалами Скарф II (5) являются точно решаемыми [16]. Эти два обстоятельства - разделение переменных и решаемость одномерных задач - позволяют полностью решить уравнение Шредингера с гамильтонианом $H^{(2)}$, т. е., как это бывает обычно в задачах с разделением переменных, аналитически найти все его собственные значения энергии и волновые функции:

$$
\Psi_{n, m}^{(2) \pm}(\vec{x})=\eta_{n}\left(x_{1}\right) \eta_{m}\left(x_{2}\right) \pm \eta_{m}\left(x_{1}\right) \eta_{n}\left(x_{2}\right),
$$

где $\eta(x)$ - известные решения одномерной задачи Скарф II:

$$
\eta_{n}(x)=(\operatorname{ch} x)^{-b} e^{-c \cdot \operatorname{arctg}(\operatorname{sh} x)} P_{n}^{(\gamma, \beta)}(i \operatorname{sh} x), \quad \gamma \equiv-\left(b+i c+\frac{1}{2}\right), \quad \beta \equiv \gamma^{\star},
$$

выраженные через полиномы Якоби [19].

В свою очередь, гамильтониан $H^{(1)}$ по-прежнему содержит смешанный член, и его спектр можно исследовать с помощью соотношений сплетания (1). А именно, все его нормируемые волновые функции

$$
\Psi_{n, m}^{(1)}(\vec{x})=Q^{+} \Psi_{n, m}^{(2)-}
$$

получаются в виде выражений через полиномы Якоби (см. формулы (9), (11), (12) в работе [18]). Из (8) исключаются симметричные функции $\Psi^{(2)+}$, поскольку они 
привели бы к ненормируемым волновым функциям $\Psi^{(1)}$. Связанные состояния с дискретными значениями энергии имеют вид

$$
\left.\varepsilon_{n, m}^{(1)}(a)\right|_{a=-1}=-(b-n)^{2}-(b-m)^{2}, \quad n, m<b, \quad|n-m|>1 .
$$

Данный результат получен для параметра со значением $a=-1$, и его можно расширить на произвольное отрицательное целое число $a_{k}=-k, k=2,3, \ldots$, за счет свойства форминвариантности: гамильтонианы-суперпартнеры $H^{(1)}, H^{(2)}$ в (4) связаны следующим образом:

$$
H^{(1)}\left(a_{k}\right)=H^{(2)}\left(a_{k+1}\right)
$$

где явно указана зависимость от параметра $a$. Для этих значений $a$ дискретный энергетический спектр $\left.\varepsilon_{n, m}(a)\right|_{a=-k}$ для $\left.H^{(1)}(a)\right|_{a=-k}$ выражается той же формулой (9), но теперь только для $|n-m|>k$. Кажущееся вырождение полученных уровней энергии относительно замены $n \rightleftarrows m$ здесь отсутствует из-за сингулярных свойств $Q^{+}$: нормируемыми являются только симметричные собственные функции $\Psi^{(1)}$. Соответствующие аналитические выражения для волновых функций $\left.\Psi^{(1)}(\vec{x} ; a)\right|_{a=-k}$ можно вывести аналитически [18] согласно (8), они явно зависят от параметра $a_{k}=-k$.

2.2. Квазиточная решаемость. Интересно сравнить полученные выше результаты с результатами, полученными для того же гамильтониана $H^{(1)}$ из (4) другим методом - второй процедурой суперсимметричного разделения переменных [13]. Этот метод работает благодаря разделению переменных в сплетающем суперзаряде $Q^{-}$. В работах [4], [12] было показано, что с помощью подходящего калибровочного (неунитарного) преобразования задачи в случае лоренцевой метрики суперзаряда разделение переменных в $Q^{-}$можно сделать при произвольных коэффициентных функциях. В частности, это возможно и для конкретных коэффициентов, имеющихся в формуле (3). Впервые эта процедура была реализована для двумерного потенциала Морса в работе [10], повторена для двумерного потенциала Пешля-Теллера в [15] и, наконец, для двумерной модели Скарф II в [20]. Мы кратко воспроизведем основные этапы этой процедуры, но для несколько другой формы потенциала. Это необходимо, чтобы сравнить данные результаты с результатами, полученными выше при значениях $a_{k}=-k$.

Мы снова начнем с тех же гамильтонианов (4), (5), удовлетворяющих соотношениям сплетания (1) с сопряженным суперзарядом $Q^{-}$, представленным в (3). Полезно совершить следующее (калибровочное) преобразование подобия:

$$
Q^{-}=e^{-\chi} q^{-} e^{+\chi}, \quad \chi(\vec{x})=-a \ln \left|\operatorname{ch} \frac{x_{+}}{2} \operatorname{sh} \frac{x_{-}}{2}\right|,
$$

чтобы разделить переменные $x_{1}, x_{2}$ в суперзаряде:

$$
q^{-}=\partial_{1}^{2}-\partial_{2}^{2}-U\left(x_{1}\right)+U\left(x_{2}\right) .
$$

Удачно, что, как и в п. 2.1, явная форма одномерных “потенциалов" $U(x)$ в $(12)$ после разделения переменных допускает аналитическое решение, что бывает довольно редко. Нуль-моды $q^{-} \omega_{n}(\vec{x})=0$ вспомогательного оператора $q^{-}$можно записать как сумму произведений:

$$
\omega_{n}(\vec{x})=\eta_{n}\left(x_{1}\right) \eta_{n}\left(x_{2}\right), \quad\left(-\partial_{1}^{2}+U(x)\right) \eta_{n}(x)=\epsilon_{n} \eta_{n}(x), \quad \epsilon_{n}=-(b-n)^{2}, \quad n<b .
$$


Решения уравнения типа Шредингера (13) известны в виде решений (7) одномерной задачи Скарф II и выражаются аналитически через полиномы Якоби. С учетом преобразования подобия (11) получаются нуль-моды $\Omega_{n}(\vec{x})$ исходного суперзаряда $Q^{-}$:

$$
\Omega_{n}(\vec{x})=e^{-\chi(\vec{x})} \omega_{n}(\vec{x})=\left|\operatorname{ch} \frac{x_{+}}{2} \operatorname{sh} \frac{x_{-}}{2}\right|^{a} \eta_{n}\left(x_{1}\right) \eta_{n}\left(x_{2}\right) .
$$

Так называемая вторая процедура суперсимметричного разделения переменных [10], [12], [13] основана на построении нормируемых нуль-мод $\Omega_{n}$, подходящие линейные комбинации которых дают некоторое подмножество (квазиточная решаемость!) нормируемых волновых функций $H^{(1)}$. Из выражения (14) следует, что эта схема возможна при любых значениях параметра $a$, но с единственным ограничением $a>-1 / 2$ : в противном случае нуль-моды $\Omega_{n}$, убывая на бесконечности, не являются $L_{2}$-интегрируемыми вдоль линии $x_{-}=0$. Таким образом, волновые функции, получаемые в рамках второго алгоритма суперсимметричного разделения переменных, соответствуют области значений параметра $a$, которая не пересекается с областью значений в первой процедуре суперсимметричного разделения переменных, где параметр $а$ принимал дискретные значения $a_{k}=-k, k=1,2, \ldots$.

Согласно второму из соотношений сплетания (1) множество нуль-мод $\Omega_{n}$ замкнуто относительно действия оператора $H^{(1)}$, т. е. действие $H^{(1)}$ на $\Omega_{n}$ дает линейную комбинацию нуль-мод $\Omega$ :

$$
H^{(1)} \Omega_{n}(\vec{x})=\sum_{k=0}^{N} c_{n k} \Omega_{k}(\vec{x})
$$

где $c_{n k}$ образуют постоянную $(N+1) \times(N+1)$-матрицу $\widehat{C}$. Можно проверить, что матрица $\widehat{C}$ треугольна, и, следовательно, ее собственные значения совпадают с диагональными элементами, которые, в свою очередь, определяют дискретный энергетический спектр $E_{n}$ гамильтониана $H^{(1)}$. По умолчанию параметр $a$ принимает фиксированные значения в области $a>-1 / 2$. Вычисление диагональных элементов $\widehat{C}$ дает

$$
c_{n, n}=E_{n, 0}=2 \epsilon_{n}-a^{2}+2 a(b-n)=-(b-n)^{2}-(b-a-n)^{2}, \quad a>-1 / 2 .
$$

Что касается волновых функций $\Psi_{n}^{(1)}(\vec{x} ; a)$, они могут быть получены аналитически путем явной диагонализации матрицы $\widehat{C}$, которая выполняется с помощью рекуррентного алгоритма [10], [12], [13], [20]. Таким образом, для этих значений параметра $a$ мы имеем дело с классом квазиточно решаемых моделей: знаем часть спектра и соответствующие волновые функции.

Свойство форминвариантности рассматриваемой модели

$$
H^{(2)}(\vec{x} ; a)=H^{(1)}(\vec{x} ; a+1)
$$

позволяет расширить множество известных волновых функций и соответствующих энергий для этой квазиточно решаемой модели. Точнее, для каждой найденной выше "основной" волновой функции $\Psi_{n, 0}^{(1)}(\vec{x} ; a+m$ ) (но с параметром $a+m$ вместо $a$ ) можно построить целое множество новых волновых функций $\Psi_{n, m}^{(1)}(\vec{x} ; a)$ с параметром $а$ следующим образом:

$$
\Psi_{n, m}^{(1)}(\vec{x} ; a)=Q^{+}(a) Q^{+}(a+1) \ldots Q^{+}(a+m-1) \Psi_{n, 0}^{(1)}(\vec{x} ; a+m)
$$


с соответствующими энергиями

$$
E_{n, m}^{(1)}(a)=E_{n, 0}^{(1)}(a+m)=-(b-n)^{2}-(b-a-m-n)^{2} .
$$

Сделаем замечание относительно обозначений. В соотношении (19) первый индекс $n$ у величины энергии нумерует порядковый номер диагонального элемента матрицы $\widehat{C}$, т. е. порядковый номер “основной” волновой функции, построенной как линейная комбинация нуль-мод $\Omega$, в то время как второй индекс $m$ нумерует количество операторов $Q^{+}$в цепочке $(18)$.

Рассматриваемая квазиточно решаемая модель обладает еще одной симметрией, которая позволяет расширить класс известных волновых функций. Мы имеем в виду дополнительную - вторую - форминвариантность системы [20]. Напомним, что гамильтониан $H^{(1)}(\vec{x})$ из (4) зависит не только от параметра $a$ (константы связи), но также от двух других $-b$ и $c$. Переформулируя кратко наблюдение, сделанное в работах [11], [20], отметим, что гамильтониан $H^{(1)}(\vec{x})$ участвует также в другой паре соотношений сплетания. Удобно идентифицировать $H^{(1)} \equiv \widetilde{H}^{(1)}$, тогда

$$
\widetilde{H}^{(1)} \widetilde{Q}^{+}=\widetilde{Q}^{+} \widetilde{H}^{(2)}, \quad \widetilde{Q}^{-} \widetilde{H}^{(1)}=\widetilde{H}^{(2)} \widetilde{Q}^{-} .
$$

Эти соотношения сплетания имеют форму, аналогичную (1), первый гамильтониан тот же, а отличие заключается в конкретных выражениях для операторов $\widetilde{Q}^{ \pm}, \widetilde{H}^{(2)}$, а именно: гамильтонианы-суперпартнеры в (20) имеют вид

$$
\begin{aligned}
\widetilde{H}^{(1),(2)}(\vec{x})=- & \Delta^{(2)}-\frac{a(a-1)}{2}\left(\frac{1}{\operatorname{ch}^{2}\left(x_{+} / 2\right)}-\frac{1}{\operatorname{sh}^{2}\left(x_{-} / 2\right)}\right)+ \\
& +\frac{c(2 b \pm 1) \operatorname{sh} x_{1}-b(b \pm 1)+c^{2}}{\operatorname{ch}^{2} x_{1}}+\frac{c(2 b \pm 1) \operatorname{sh} x_{2}-b(b \pm 1)+c^{2}}{\operatorname{ch}^{2} x_{2}}
\end{aligned}
$$

и сплетаются посредством новых суперзарядов

$$
\begin{aligned}
\widetilde{Q}^{ \pm}=\partial_{1} \partial_{2} & \mp\left(b \operatorname{th} x_{1}+\frac{c}{\operatorname{ch} x_{1}}\right) \partial_{2} \mp\left(b \operatorname{th} x_{2}+\frac{c}{\operatorname{ch} x_{2}}\right) \partial_{1}+ \\
+ & \left(b \operatorname{th} x_{1}+\frac{c}{\operatorname{ch} x_{1}}\right)\left(b \operatorname{th} x_{2}+\frac{c}{\operatorname{ch} x_{2}}\right)+\frac{a(a-1)}{4 \operatorname{ch}^{2}\left(x_{+} / 2\right)}+\frac{a(a-1)}{4 \operatorname{sh}^{2}\left(x_{-} / 2\right)} .
\end{aligned}
$$

Важное обстоятельство состоит в том, что новый гамильтониан $\widetilde{H}^{(2)}$ не совпадает с исходным $H^{(2)}$. Легко убедиться, что пара $\widetilde{H}^{(1)} \equiv H^{(1)}$ и $\widetilde{H}^{(2)}$ также обладает свойством форминвариантности, но теперь относительно параметра $b$ :

$$
H^{(1)}(\vec{x} ; a, b)=\widetilde{H}^{(2)}(\vec{x} ; a, b+1) .
$$

По аналогии с предыдущей процедурой сплетания (20) позволяют построить новые волновые функции $\widetilde{\Psi}^{(1)}$, начиная с произвольной “основной” волновой функции. Роль этого “основного" состояния может выполнять волновая функция $\Psi_{n, m}^{(1)}(\vec{x} ; a, b)$, полученная выше в (18). Таким образом, у нас имеется множество волновых функций гамильтониана $H^{(1)}(\vec{x} ; a, b)$, которые состоят из произведения некоторого количества операторов $\widetilde{Q}^{+}$и некоторого числа операторов $Q^{+}$, действующих на "основную" функцию $\Psi_{n, 0}^{(1)}(\vec{x})$ :

$$
\begin{aligned}
& \Psi_{n, m, M}^{(1)}(\vec{x} ; a, b)=\widetilde{Q}^{+}(a, b) \widetilde{Q}^{+}(a, b-1) \ldots \widetilde{Q}^{+}(a, b-M+1) \Psi_{n, m}^{(1)}(\vec{x} ; a, b-M)= \\
&=\widetilde{Q}^{+}(a, b) \widetilde{Q}^{+}(a, b-1) \ldots \widetilde{Q}^{+}(a, b-M+1) Q^{+}(a, b-M) \times \\
& \quad \times Q^{+}(a+1, b-M) \ldots Q^{+}(a+m-1, b-M) \Psi_{n, 0}^{(1)}(\vec{x} ; a+m, b-M)
\end{aligned}
$$


где индекс $M$ обозначает число операторов $\widetilde{Q}^{+}$. Положение операторов $\widetilde{Q}^{+}$и $Q^{+}$ в этой цепочке может быть изменено посредством соотношения

$$
\widetilde{Q}^{+}(\vec{x} ; a, b) Q^{+}(\vec{x} ; a, b-1)=Q^{+}(\vec{x} ; a, b) \widetilde{Q}^{+}(\vec{x} ; a+1, b),
$$

обе стороны этого равенства сплетают одну и ту же пару гамильтонианов: $H^{(1)}(\vec{x} ; a, b)$ и $H^{(1)}(\vec{x} ; a+1, b-1)$.

\section{3. ИНТЕГРИРУЕМОСТЬ СИСТЕМЫ}

Можно проверить [4], что система с гамильтонианом $H^{(1)}$, удовлетворяющим соотношениям сплетания, имеет оператор симметрии $R^{(1)}$ четвертого порядка по производным, и его явный вид известен в терминах суперзарядов:

$$
R^{(1)}=Q^{+} Q^{-}, \quad\left[H^{(1)}, R^{(1)}\right]=0 .
$$

Данное свойство симметрии гамильтониана является общим следствием соотношений сплетания: любой эрмитов оператор, сплетаемый с любым другим оператором посредством операторов $Q^{ \pm}$, коммутирует с соответствующим оператором симметрии $R^{(1)}$. Между тем известно [21], что для любой двумерной системы взаимно коммутирующих операторов симметрии не может быть два или более, т. е. $H^{(1)}$ описывает вполне интегрируемую модель.

Найденный ранее спектр (19) невырожден, и потому все волновые функции (18) гамильтониана $H^{(1)}$ должны быть и собственными функциями $R^{(1)}$. Это свойство не очевидно следует из явной формы (18) с произвольным числом множителей $Q^{+}(a+k)$. С этой точки зрения было бы интересно проверить данное свойство непосредственно. Это особенно интересно, поскольку в случае двумерного потенциала Морса [10], кроме волновых функций, аналогичных (18), были "вручную" построены три дополнительные волновые функции, и непосредственным вычислением было показано, что каждая из них является собственной функцией оператора симметрии. Кроме того, в нашем случае для некоторых значений констант связи возможно случайное вырождение спектра. А именно, в соответствии с (19) два энергетических уровня $E_{n, m}$ и $E_{n^{\prime}, m^{\prime}}$ для волновых функций (18) могут совпасть для модели со специальным соотношением между двумя параметрами $a$ и $b$ :

$$
\begin{aligned}
a(\delta m-\delta n)+b(2 \delta n+\delta m) & =\left(n+n^{\prime}\right) \delta n+1 / 2\left(m+m^{\prime}\right) \delta m+\left(n m-n^{\prime} m^{\prime}\right), \\
\delta n & \equiv n-n^{\prime}, \quad \delta m \equiv m-m^{\prime}
\end{aligned}
$$

Вообще при выполнении этого соотношения действие оператора симметрии $R^{(1)}$ на функции $\Psi_{n, m}^{(1)}$ может перевести ее в другую функцию $\Psi_{n^{\prime}, m^{\prime}}^{(1)}$ с той же энергией. Разумеется, эта проблема существует также для более общей волновой функции, полученной из произведения $Q^{+}$и $\widetilde{Q}^{+}$.

Итак, необходимо вычислить результат действия оператора симметрии на произвольную волновую функцию данного вида. Стандартным методом математической индукции докажем, что произвольная собственная функция (24) гамильтониана $H^{(1)}$ одновременно является собственной функцией оператора симметрии $R^{(1)}$. Для простоты начнем с функций вида (18). Прежде всего, существует соотношение между $R^{(1)}(a)$ и $R^{(2)}(a-1)$. Благодаря форминвариантности (17) оба оператора $R^{(1)}(a)$ и $R^{(2)}(a-1)$ являются операторами симметрии для одного и того же 
гамильтониана $H^{(1)}(a)$, и поэтому они должны совпадать с точностью до функции от самого гамильтониана $H^{(1)}(a)$. Фактически единственная возможность:

$$
R^{(1)}(a)=R^{(2)}(a-1)+\alpha(a) H^{(1)}(a)+\beta(a),
$$

где опущена зависимость $R$ и $H$ от $\vec{x}$, а $\alpha(a), \beta(a)$ не зависят от $\vec{x}$. Явно коэффициенты $\alpha, \beta$ были вычислены в работе [11] (см. уравнение после (34) в [11]):

$$
\alpha(a)=2(2 a-1), \quad \beta(a)=(2 a-1)\left(2 a^{2}-2 a-3\right) .
$$

Соотношение, аналогичное $(28)$, связывает операторы $\widetilde{R}^{(1)}(a, b)$ и $\widetilde{R}^{(2)}(a, b+1)$.

Теперь для начала доказательства методом индукции (зависимость от $b$ опущена) запишем

$$
\begin{aligned}
& R^{(1)}(a) \Psi_{n, 1}^{(1)}(\vec{x} ; a)=Q^{+}(a) Q^{-}(a) Q^{+}(a) \Psi_{n, 0}^{(1)}(\vec{x} ; a+1)= \\
& \quad=Q^{+}(a)\left(Q^{+}(a+1) Q^{-}(a+1)-\alpha(a+1) E_{n, 0}^{(1)}(a+1)-\beta(a+1)\right) \Psi_{n, 0}^{(1)}(\vec{x} ; a+1)= \\
& \quad=-\left(E_{n, 1}^{(1)}(a) \alpha(a+1)+\beta(a+1)\right) \Psi_{n, 1}^{(1)}(\vec{x} ; a),
\end{aligned}
$$

где использовано явное выражение (19), что доказывает утверждение для $m=1$. Случай $m=2$ также можно аналогично вычислить, а результат приводит к гипотезе для высших $m$. Предположим, для общего значения $m$ выполняется соотношение

$$
R^{(1)}(a) \Psi_{n, m}^{(1)}(\vec{x} ; a)=-\left(\sum_{k=1}^{m}\left[E_{n, m}^{(1)}(a) \alpha(a+k)+\beta(a+k)\right]\right) \Psi_{n, m}^{(1)}(\vec{x} ; a) .
$$

Для второго шага индукции имеем

$$
\begin{aligned}
& R^{(1)}(a) \Psi_{n, m+1}^{(1)}(\vec{x} ; a)=R^{(1)}(a) Q^{+}(a) \Psi_{n, m}^{(1)}(\vec{x} ; a+1)= \\
& \quad=Q^{+}(a)\left(R^{(1)}(a+1)-\alpha(a+1) E_{n, m}^{(1)}(a+1)-\beta(a+1)\right) \Psi_{n, m}^{(1)}(\vec{x} ; a+1)= \\
& \quad=-\left(\sum_{k=1}^{m+1}\left[E_{n, m}^{(1)}(a+1) \alpha(a+k)+\beta(a+k)\right]\right) \Psi_{n, m+1}^{(1)}(\vec{x} ; a)= \\
& \quad=-\left(\sum_{k=1}^{m+1}\left[E_{n, m+1}^{(1)}(a) \alpha(a+k)+\beta(a+k)\right]\right) \Psi_{n, m+1}^{(1)}(\vec{x} ; a) .
\end{aligned}
$$

Тем самым утверждение доказано: волновые функции вида (18) являются одновременно собственными функциями оператора симметрии $R^{(1)}$, независимо от возможной случайной вырожденности энергетического уровня.

Аналогично волновые функции (24), полученные цепочкой операторов $\widetilde{Q}^{+}$и $Q^{+}$, тоже являются собственными функциями $R^{(1)}$. В этом случае доказательство основано на соотношении между двумя различными формами оператора симметрии для одного и того же гамильтониана $H^{(1)}$ :

$$
R^{(1)}=Q^{+} Q^{-}, \quad \widetilde{R}^{(1)}=\widetilde{Q}^{+} \widetilde{Q}^{-} .
$$

Оба оператора имеют один и тот же (четвертый) порядок по производным, а для двумерной системы не может существовать никакого второго - в дополнение к $R^{(1)}-$ независимого оператора симметрии. Такое возможно, только если оба оператора 
в (33) совпадают с точностью до полинома второго порядка от гамильтониана $H^{(1)}$ с постоянными коэффициентами. Из-за довольно громоздкого характера выражений для операторов в (33) коэффициенты были определены с помощью аналитических вычислений с помощью программы Mathematica [22]:

$$
R^{(1)}(a, b)=-4 \widetilde{R}^{(1)}(a, b)+\left(H^{(1)}\right)^{2}+\left(2 a^{2}+4 b^{2}\right) H^{(1)}+\left(a^{4}+4 b^{4}\right) .
$$

В отличие от соотношения (28), соотношение между $\widetilde{R}^{(1)}$ и $R^{(1)}$ не имеет отношения к форминвариантности.

Для примера рассмотрим действие оператора симметрии $\widetilde{R}^{(1)}(a, b)$ на $\Psi_{n, m, 1}^{(1)}(\vec{x} ; a, b)$, т. е. $M=1$ в (24):

$$
\begin{aligned}
& \widetilde{R}^{(1)}(a, b) \Psi_{n, m, 1}^{(1)}(\vec{x} ; a, b)=\widetilde{R}^{(1)}(a, b) \widetilde{Q}^{+}(a, b) \Psi_{n, m}^{(1)}(\vec{x} ; a, b-1)= \\
& =\widetilde{Q}^{+}(a, b) \widetilde{R}^{(2)}(a, b) \Psi_{n, m}^{(1)}(\vec{x} ; a, b-1)= \\
& =\widetilde{Q}^{+}(a, b)\left(\widetilde{R}^{(1)}(a, b-1)+\widetilde{\alpha}(a, b) H^{(1)}(a, b-1)+\widetilde{\beta}(a, b)\right) \Psi_{n, m}^{(1)}(\vec{x} ; a, b-1)= \\
& =\left(\widetilde{\alpha}(a, b) E_{n, m}^{(1)}(a, b-1)+\widetilde{\beta}(a, b)\right) \Psi_{n, m, 1}^{(1)}(\vec{x} ; a, b)+ \\
& \quad+\widetilde{Q}^{+}(a, b) \widetilde{R}^{(1)}(a, b-1) \Psi_{n, m}^{(1)}(\vec{x} ; a, b-1) .
\end{aligned}
$$

Последнее слагаемое вычисляется с помощью (34):

$$
\begin{aligned}
& \widetilde{Q}^{+}(a, b) \widetilde{R}^{(1)}(a, b-1) \Psi_{n, m}^{(1)}(\vec{x} ; a, b-1)=\widetilde{Q}^{+}(a, b)\left(R^{(1)}(a, b-1)-\right. \\
& \left.\quad-c_{1}\left(H^{(1)}(a, b-1)\right)^{2}-c_{2} H^{(1)}(a, b-1)-c_{3}\right) \Psi_{n, m}^{(1)}(\vec{x} ; a, b-1)= \\
& =\text { const } \cdot \Psi_{n, m, 1}^{(1)}(\vec{x} ; a, b) .
\end{aligned}
$$

Таким образом,

$$
\widetilde{R}^{(1)}(a, b) \Psi_{n, m, 1}^{(1)}(\vec{x} ; a, b)=\text { const } \cdot \Psi_{n, m, 1}^{(1)}(\vec{x} ; a, b) .
$$

Случай произвольного $M>1$ может быть доказан аналогично.

\section{4. ЗАКЛЮЧЕНИЕ}

Настоящая работа продолжает исследование двумерной интегрируемой модели Скарф II, которая не допускает процедуры обычного разделения переменных. В частности, исследованы зависимость спектра от параметра модели, его возможное вырождение и свойства оператора симметрии четвертого порядка. Аналогичные свойства двух других двумерных моделей, не допускающих разделения переменных, моделей Морса и Пешля-Теллера - могут быть изучены тем же путем. Например, двумерная система Морса [10], [14] точно решается при параметре $a=-(k+1) / 2$, где $k=0,1,2, \ldots$, и квазиточно в непересекающейся области $a>1 / 4+1 / 4 \sqrt{2}$.

Благодарности. Работа частично поддержана Санкт-Петербургским государственным университетом (гранты № 11.38.223.2015 (Е.В. Колеватова), № 11.42. 1303.2014 (М. В. Иоффе)) и РФФИ (грант № 13-01-00136_а (М. В. Иоффе, Е. В. Колеватова)). 


\section{Список литературы}

[1] F. Cooper, A. Khare, U. Sukhatme, Phys. Rep., 251:5-6 (1995), 267-385, arXiv: hep-th/9405029; G. Junker, Supersymmetric Methods in Quantum and Statistical Physics, Springer, Berlin, 1996; B. K. Bagchi, Supersymmetry in Quantum and Classical Mechanics, Chapman, Boca Raton, FL, 2001; J. D. Fernández C, AIP Conf. Proc., 1287 (2010), 3-36, arXiv: 0910.0192; A. A. Andrianov, M. V. Ioffe, J. Phys. A: Math. Theor., 45:50 (2012), 503001, 62 pp., arXiv: 1207.6799.

[2] А. А. Андрианов, Н. В. Борисов, М. В. Иоффе, Писъма в ЖЖЭФ, 39:2, 78-81; ТМФ, 61:2 (1984), 183-198; A. A. Andrianov, N. V. Borisov, M. V. Ioffe, Phys. Lett. A, 105:1-2 (1984), 19-22; A. A. Andrianov, N. V. Borisov, M. I. Eides, M. V. Ioffe, Phys. Lett. A, 109:4 (1985), 143-148; А. А. Андрианов, Н. В. Борисов, М. В. Иоффе, М. И. Эйдес, $T M \Phi, \mathbf{6 1 : 1}(1984), 17-28$.

[3] A. A. Andrianov, M. V. Ioffe, Phys. Lett. B, 205:4 (1988), 507-510; M. V. Ioffe, A. I. Neelov, J. Phys. A: Math. Gen., 36:10 (2003), 2493-2506, arXiv: hep-th/0302004.

[4] A. A. Andrianov, M. V. Ioffe, D. N. Nishnianidze, Phys. Lett. A, 201:1 (1995), 103-110, arXiv: hep-th/9404120; "Higher order SUSY in quantum mechanics and integrability of two-dimensional Hamiltonians", arXiv: solv-int/9605007; J. Phys. A: Math. Gen., 32:25 (1999), 4641-4654; А. А. Андрианов, М. В. Иоффе, Д. Н. Нишнианидзе, ТМФ, 104:3 (1995), 463-478.

[5] M. S. Bardavelidze, M. V. Ioffe, D. N. Nishnianidze, Phys. Lett. A, 377:3-4 (2013), 195-199, arXiv: 1211.1461.

[6] Л.Э. Генденшгтейн, Писъма в ЖЭТФ, 38:6 (1983), 299-302.

[7] A. A. Andrianov, F. Cannata, M. V. Ioffe, D. N. Nishnianidze, Phys. Lett. A, 266:4-6 (2000), 341-349; J. Bougie, A. Gangopadhyaya, J. V. Mallow, Phys. Rev. Lett., 105:21 (2010), 210402, 4 pp.

[8] J.W. Dabrowska, A. Khare, U.P. Sukhatme, J. Phys. A: Math. Gen., 21:4 (1988), L195-L200.

[9] C. Quesne, J. Phys. A: Math. Theor., 41:39 (2008), 392001, 6 pp.; F. Cannata, M. V. Ioffe, E. V. Kolevatova, D. N. Nishnianidze, Ann. Phys., 356 (2015), 438-451.

[10] F. Cannata, M. V. Ioffe, D. N. Nishnianidze, J. Phys. A: Math. Gen., 35 (2002), 1389-1404.

[11] F. Cannata, M. V. Ioffe, D. N. Nishnianidze, J. Math. Phys., 52:2 (2011), 022106, 9 pp.

[12] M. V. Ioffe, J. Phys. A: Math. Gen., 37:43 (2004), 10363-10374.

[13] M. V. Ioffe, SIGMA, 6 (2010), 075, 10 pp., arXiv: 1009.4764.

[14] M. V. Ioffe, D. N. Nishnianidze, Phys. Rev. A, 76:5 (2007), 052114, 5 pp.

[15] M. V. Ioffe, P. A. Valinevich, J. Phys. A: Math. Gen., 38:11 (2005), 2497-2510, arXiv: hep-th/0409153; M. V. Ioffe, D. N. Nishnianidze, P. A. Valinevich, J. Phys. A: Math. Theor., 43:48 (2010), 485303, 17 pp.

[16] F. L. Scarf, Phys. Rev., 112:4 (1958), 1137-1140.

[17] A.V. Turbiner, Commun. Math. Phys., 118:3 (1988), 467-474; A. G. Ushveridze, Quasi-Exactly Solvable Problems in Quantum Mechanics, Institute of Physics Publishing, Bristol, 1994; M. A. Shifman, A. V. Turbiner, Commun. Math. Phys., 126:2 (1989), $347-365$.

[18] M. V. Ioffe, E. V. Krupitskaya, D. N. Nishnianidze, Ann. Phys., 327:3 (2012), 764-773.

[19] Г. Бейтмен, А. Эрдейи, Высшие траниендентные функиии, т. 2: Функиии Бесселя, функиии параболического иилиндра, ортогональные многочлены, Наука, М., 1974.

[20] M. V. Ioffe, E. V. Krupitskaya, D. N. Nishnianidze, Europhys. Lett., 98:1 (2012), 10013, 6 pp., arXiv: 1203.3076.

[21] W. Miller Jr., Symmetry and Separation of Variables, Addison-Wesley, London, 1977.

[22] S. Wolfram, Mathematica. Ver. 10.0, Wolfram Research, Champaign, IL, 2014. 